\title{
The continuous structure bending of the three- layer plate with the lightweight aggregate
}

\author{
Alexander Zhuravlev ${ }^{1}$, Dmitry Zhuravlev ${ }^{1 *}$ and Anastasia Melnik ${ }^{1}$ \\ ${ }^{1}$ Don State Technical University, 344022, Sotcialisticheskaya, 162, Rostov-on-Don, Russia
}

\begin{abstract}
This paper deals with the problem of finding the effective way to solve the task of the composite construction bending of the three-layer plate with the lightweight aggregate, which operates on a continuous pattern. The expressions for the parameters determination, regarding a reduction of support bending moments due to the shear deformation of the middle layer, are given. The three-membered structure equation, connecting three bending moments, occurring above three adjacent pillars of the continuous three-layer beam, is obtained based on the condition of the movement compatibility.
\end{abstract}

\section{Introduction}

The increasing application of the composite constructions has been found recently in many technical fields and, in particular, they are used in the protecting parts of the buildings' and constructions' covering in the form of the three-layer plates with the lightweight aggregate. The translucent dome roof made of three-layer plates with the sheet glass-reinforced plastic cladding and the plastic ring middle layer can illustrate one of such composite constructions [1]. The paper [2] describes the procedure of the hybrid matrix construction of a three-layer element stiffness, which is represented by a selected on the multi-faceted dome surface triangular panel in the form of an oblique triangle. The issues of the local instability, connected with the faces creasing of the outer layers of the triangular and hexagonal three-layer plates, are investigated in the works [3], [4].

The general theory of the three-layer plate bending is described in detail in the works [5], [6]. Vibration and stability problems of three-layer structures are represented in the works [7], [8] and [9]. The work [10] is devoted to the approximate approach of the threelayer structures calculation taking into consideration the rheological peculiarities of the aggregate.

\section{Problem definition}

The three-layer elements of the covering with the sheet steel cladding have a width of $1000 \mathrm{~mm}$ and a cladding thickness within from 0.4 to $0.75 \mathrm{~mm}$. They can be up to $26.5 \mathrm{~m}$ in length. The thickness of the middle layer is from 20 to $100 \mathrm{~mm}$ [11].

\footnotetext{
*Corresponding author: rodjer1980@mail.ru
} 
The thread-cutting screws are widely used to connect thin steel claddings in the case of longitudinal joints. These thread-cutting screws have additional threading on the back side, in the result of which the packing washer compression on the upper cladding is achieved. The outer claddings of the adjacent elements are overlapped with each other along a longitudinal seam and are interconnected by the screws approximately every $50 \mathrm{~cm}$. The cross joints should be avoided, using the extended threelayer constructions.

\section{Results and discussion}

In this regard the three-layer plate construction of the covering as a beam with nine spans of the equal length $l=2.0 \mathrm{~m}$ is under our investigation. Fig. 1 shows the scheme of such threelayer construction, which is successfully applied in the sports hall building covering [12].

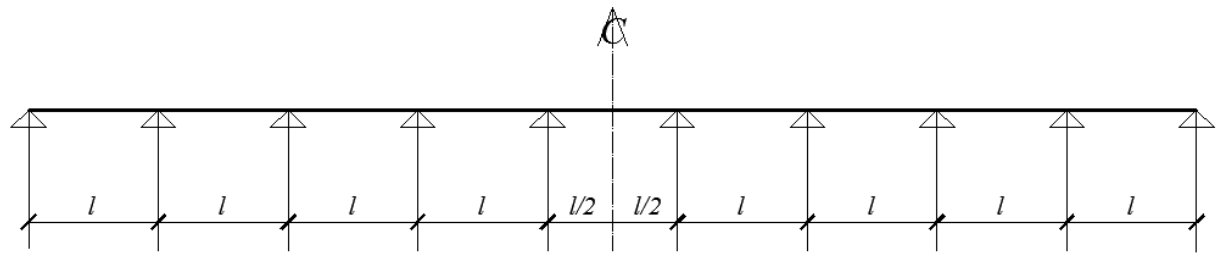

Fig. 1. The continuous three-layer beam scheme

Due to the system symmetry and the actual loading on it we discuss the left half of the multi-span continuous construction of the three-layer beam up to the symmetry line (Fig. 2).

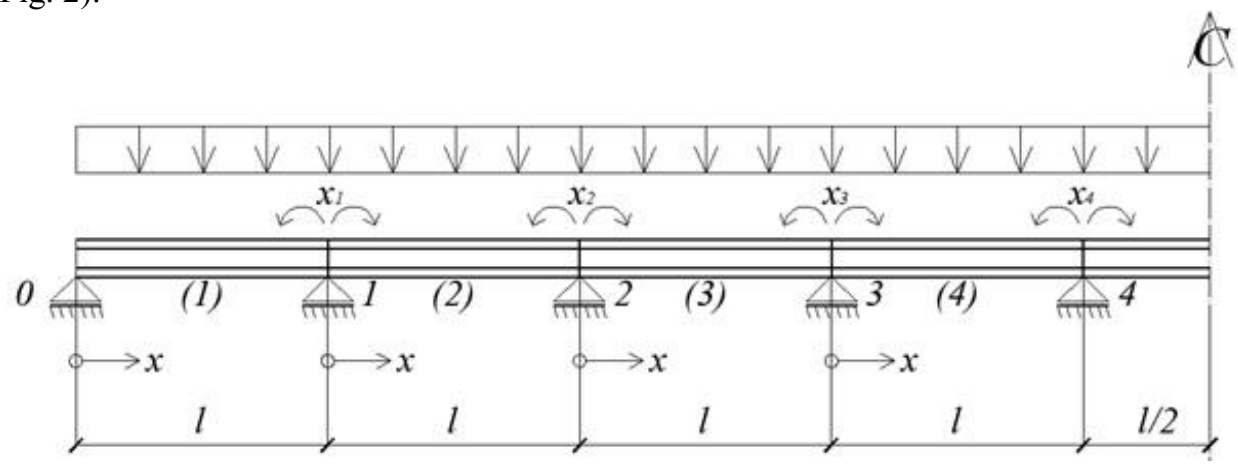

Fig. 2. The three-layer beam design scheme

The behavior of the three-layer beam with the lightweight aggregate, having very thin claddings, is described by the following system of the differential equations [13]:

$$
\begin{aligned}
& D \frac{d^{2} u}{d x^{2}}-G_{3}\left(\frac{2}{H} u+\frac{d w}{d x}\right)=0, \\
& G_{3}\left(\frac{2}{H} \frac{d u}{d x}+\frac{d^{2} w}{d x^{2}}\right)=-\frac{q}{H}
\end{aligned}
$$

After having solved this system of equations we are provided with [14] : 


$$
\begin{aligned}
& \frac{d u}{d x}=-\frac{q x^{2}}{2 H D}+C_{1} x+C_{2} ; \quad u=-\frac{q x^{3}}{6 H D}+C_{1} \frac{x^{2}}{2}+C_{2} x+C_{3}, \\
& w=-\frac{q x^{2}}{2 G_{3} H}+\frac{2}{H}\left[\frac{q x^{4}}{24 H D}-C_{1}\left(\frac{x^{3}}{6}-\frac{H D x}{2 G_{3}}\right)-C_{3} x\right]+C_{4}
\end{aligned}
$$

In the expressions (1)-(4) under $\mathrm{u}$ we understand the movement in the upper cladding of the three-layer beam, and under $w$ - its deflection. The first condition (3) implies that under $x=0: u^{\prime}(0)=0 ; w(0)=0$. Substituting the expressions (3) and (4) in these conditions, we obtain $C_{2}=0$ and $C_{4}=0$. Consequently, we conclude

$$
\begin{aligned}
& u^{\prime}=-\frac{q x^{2}}{2 H D}+C_{1} x \\
& w=-\frac{q x^{2}}{2 G_{3} H}+\frac{2}{H}\left[\frac{q x^{4}}{24 H D}-C_{1}\left(\frac{x^{3}}{6}-\frac{H D x}{2 G_{3}}\right)-C_{3} x\right] .
\end{aligned}
$$

The constant integrations $C_{1}$ and $C_{3}$ can be obtained from the following conditions at the section $x=l \quad w(l)=0 ; M(l)=X_{1} 1$. Therefore

$$
\begin{aligned}
& C_{1}=\frac{1}{H D}\left(\frac{X_{1}}{l}+\frac{q l}{2}\right), \\
& C_{3}=-\frac{1}{H D}\left[\frac{q l^{3}}{24}+\frac{X_{1}}{l}\left(\frac{l^{2}}{6}-\frac{H D}{2 G_{3}}\right)\right] .
\end{aligned}
$$

It should be mentioned that under $x=l$ the expression $u(l)$ is written in the following way:

$$
u(l)=\frac{1}{H D}\left[\frac{q l^{3}}{24}+\frac{X_{1}}{l}\left(\frac{l^{2}}{3}+\frac{H D}{2 G_{3}}\right)\right] .
$$

Magnified $H$ times shifting of the middle plane points of the upper cladding of the threelayer beam at the section 1 under $x=l$ is equal

$$
H u(l)=H u_{1}^{l}=\frac{q l^{3}}{24 D}+\frac{X_{1} l}{3 D}(1+3 \omega),
$$

where the parameter $\omega$ means

$$
\omega=\frac{H D}{2 G_{3} l^{2}}
$$

At the second part of the beam at the section we have $x=0: w(0)=0 ; M(0)=X_{1}$. In this case every time the first condition assures us that $C_{4}$ vanishes. Using the second condition we are provided with 


$$
C_{2}=\frac{1}{H D} X_{1}
$$

At the section $\mathrm{x}=1$ it is supposed to be $w(l)=0 ; M(l)=X_{2}$, whereby we have

$$
\begin{gathered}
C_{1}=\frac{1}{H D}\left(\frac{q l}{2}-\frac{X_{1}-X_{2}}{l}\right), \\
C_{3}=-24 \frac{q l^{3}}{H D}-\frac{l}{6 H D}\left(2 X_{1}+X_{2}\right)-\frac{l}{H D}\left(X_{1}-X_{2}\right) \omega .
\end{gathered}
$$

Thus, at the support section at the point 1 there is $u(0)=C_{3}=u_{1}^{r}$. From this we can conclude that

$$
H u_{1}^{r}=-\frac{l}{2 D}\left[\frac{q l^{2}}{12}+\frac{1}{3}\left(2 X_{1}+X_{2}\right)+2 \omega\left(X_{1}-X_{2}\right)\right] .
$$

The continuity condition at the point 1 is recorded as $u_{1}^{l}=u_{1}^{r}$ and in doing so we are provided with

$$
4(1+3 u) X_{1}+(1-6 \omega) X_{2}=-\frac{q l^{2}}{2} .
$$

At the second part at the point 2 under $x=l$ we have

$$
u_{2}^{l}=\frac{l}{H D}\left[\frac{q l^{2}}{24}+\frac{X_{1}+2 X_{2}}{6}-\left(X_{1}-X_{2}\right) \omega\right] .
$$

At the third part the bounding conditions are $: w(0)=0 ; M(0)=X_{2}$ uder $x=0$. Under $x=l$ it is supposed to be $w(l)=0 ; M(l)=X_{3}$. Due to these conditions it should be that

$$
\begin{aligned}
& C_{1}=\frac{1}{H D}\left(\frac{q l}{2}-\frac{X_{2}-X_{3}}{l}\right) ; \quad C_{2}=\frac{1}{H D} X_{2}, \\
& C_{3}=-\frac{l}{H D}\left[\frac{q l^{2}}{24}+\frac{2 X_{2}+X_{3}}{6}+\omega\left(X_{2}-X_{3}\right)\right] .
\end{aligned}
$$

Re-recording the conditions of the movement compatibility as $u_{2}^{l}=u_{2}^{r}$, we find out

$$
(1-6 \omega) X_{1}+4(1+3 \omega) X_{2}+(1-6 \omega) X_{3}=-\frac{q l^{2}}{2} .
$$

The bending moment for the continuous beam with central prop, occurring at the section above the middle interior support, turns out to be equal to 


$$
X_{1}=-\frac{q l^{2}}{8}\left(1-k_{1}\right)
$$

Here the parameter $k_{1}$, regarding the decrease of the support bending moment due to the shear deformation of the middle layer, is equal to

$$
k_{1}=\frac{3 \omega}{1+3 \omega},
$$

Actually the inferior index of the parameter $\mathrm{k}$ corresponds to the number of the intermediate supports in the continuous beam.

It is obvious that in the case of the three-span beam in the equation (20) it should be taken $X_{1}=X_{2}$ and $X_{3}=0$. At the same time we find that

$$
X_{1}=X_{2}=-\frac{q l^{2}}{10}\left(1-k_{2}\right),
$$

Where the parameter $k_{2}$ is defined by the formula

$$
k_{2}=\frac{6 \omega}{5+6 \omega} \text {. }
$$

It is not difficult to be sure that this formula coincides exactly with the expression for $k$, given in the work [15]. The equation (20) connects three bending moments, appearing at the sections above three adjacent supports of the continuous three-layer beam. In our case this equation, composed in sequence for each of four interior supports, gives

$$
\left[\begin{array}{cccc}
4(1+3 \omega) & 1-6 \omega & 0 & 0 \\
1-6 \omega & 4(1+3 \omega) & 1-6 \omega & 0 \\
0 & 1-6 \omega & 4(1+3 \omega) & 1-6 \omega \\
0 & 0 & 1-6 \omega & 5+6 \omega
\end{array}\right]\left[\begin{array}{c}
X_{1} \\
X_{2} \\
X_{3} \\
X_{4}
\end{array}\right]=-\frac{q l^{2}}{2}\left[\begin{array}{l}
1 \\
1 \\
1 \\
1
\end{array}\right] .
$$

The coefficients' matrix of the obtained system of four linear algebraic equations, as it can be seen, has three-membered structure. After solving this system of equations we find the bounding moments as

$$
\begin{aligned}
& X_{1}=-\frac{q l^{2}}{2} \frac{8(1+3 \omega)\left(7+60 \omega+36 \omega^{2}\right)}{(5+6 \omega)\left[54\left(1+6 \omega^{2}\right)-(1-6 \omega)^{3}\right]}, \\
& X_{2}=-\frac{q l^{2}}{2} \frac{8(1+3 \omega)(5+6 \omega)(1+12 \omega)+(1-6 \omega)^{3}}{(5+6 \omega)\left[54\left(1+6 \omega^{2}\right)-(1-6 \omega)^{3}\right]}, \\
& X_{3}=-\frac{q l^{2}}{2} \frac{4(1+3 \omega)\left(11+121 \omega+180 \omega^{2}\right)+(1+6 \omega)^{3}}{(5+6 \omega)\left[54\left(1+6 \omega^{2}\right)-(1-6 \omega)^{3}\right]}, \\
& X_{4}=-\frac{q l^{2}}{2} \frac{4(1+3 \omega)\left[12(1+6 \omega)(1+3 \omega)-(1+6 \omega)^{2}\right]}{(5+6 \omega)\left[54\left(1+6 \omega^{2}\right)-(1-6 \omega)^{3}\right]} .
\end{aligned}
$$


The expression (26) can be recorded in the following way:

$$
X_{1}=-\frac{28 q l^{2}}{265}\left(1-k_{8}^{(1)}\right)
$$

where the parameter $k_{8}^{(1)}$, taking into account the effect of the shear deformation in the aggregate of the three-layer beam, is equal to

$$
k_{8}^{(1)}=\frac{3 \omega\left(1357+11664 \omega+18684 \omega^{2}+3024 \omega^{3}\right)}{7(5+6 \omega)\left[54\left(1+6 \omega^{2}\right)-(1-6 \omega)^{3}\right]} .
$$

Having made a similar transformation of the expression (27), we are provided with

$$
X_{2}=-\frac{41 q l^{2}}{530}\left(1-k_{8}^{(2)}\right) \text {. }
$$

Here the parameter $k_{8}^{(2)}$ is equal to

$$
k_{8}^{(2)}=-\frac{6 \omega\left(2897+10134 \omega-15876 \omega^{2}-8856 \omega^{3}\right)}{41(5+6 \omega)\left[54\left(1+6 \omega^{2}\right)-(1-6 \omega)^{3}\right]} .
$$

We have for the bending moment at the section above the third interior support the following

$$
X_{3}=-\frac{9 q l^{2}}{106}\left(1-k_{8}^{(3)}\right)
$$

at this time the parameter $k_{8}^{(3)}$ is equal here to

$$
k_{8}^{(3)}=-\frac{2 \omega\left(569-1128 \omega+2916 \omega^{2}+5832 \omega^{3}\right)}{9(5+6 \omega)\left[54\left(1+6 \omega^{2}\right)-(1-6 \omega)^{3}\right]} \text {. }
$$

Since the calculation of three-layer plate with the lightweight aggregate can be restricted only by its two first parts, such transformation for the support bending moments $X_{3}$ and $X_{4}$ are unnecessary. Using the expressions for the bending moments at the sections above the first two interior supports, we define the reactions at the points 0.1 and 2 . Thus, we obtain:

$$
\begin{aligned}
& R_{0}=\frac{q l}{530}\left(209+56 k_{8}^{(1)}\right), \\
& R_{1}=\frac{q l}{530}\left[601-\left(112 k_{8}^{(1)}-41 k_{8}^{(2)}\right)\right],
\end{aligned}
$$




$$
R_{2}=\frac{q l}{530}\left(520+56 k_{8}^{(1)}-82 k_{8}^{(2)}+45 k_{8}^{(3)}\right) .
$$

Now let us assume that the three-layer construction of the plate is characterized by the symmetrical structure, and whereby the thickness of its cladding is $\mathrm{t}=0.05 \mathrm{~cm}$. The aggregate of three-layer plate is considered to have a long-term shear modulus $G_{3}$ $=40 \mathrm{kgf} / \mathrm{cm} 2$ under the volume weight $100 \mathrm{~kg} / \mathrm{m} 3$.

The aggregate thickness is $\mathrm{H}=10 \mathrm{~cm}$. The stiffness of the outer layers of the plate at tension is $\mathrm{D}=113187 \mathrm{kgf} / \mathrm{cm}$. The dimensionless parameter $\omega$ in this case is

$$
\omega=\frac{H D}{2 G_{3} l^{2}}=\frac{10 \cdot 113187}{2 \cdot 40 \cdot 200^{2}}=0,35 .
$$

The calculations for $k_{8}^{(1)}$ and $k_{8}^{(2)}$ in accordance with the expressions (31) and (33) give:

$$
k_{8}^{(1)}=0,3191 ; \quad k_{8}^{(2)}=0,0571 .
$$

Further based on the expressions (26) and (27) we get $X_{1}=-0,0719 q l^{2}$; $X_{2}=-0,0818 q l^{2}$.

These numerical values of the bending moments indicate that the upper-range value of the support moment occurs at the section already not above the first interior support as usual, but above the second interior support of the three-layer construction.

Normal stresses in the claddings of the three-layer construction, located under the influence of the proportionally distributed load $\mathrm{q}=160 \mathrm{kgf} / \mathrm{cm} 2$, is calculated by the formula

$$
\sigma_{x x}=\frac{41 q l^{2}}{530 H t}\left(1-k_{8}^{(2)}\right)=\frac{41 \cdot 0,016 \cdot 200^{2}}{530 \cdot 10 \cdot 0,05}(1+0,0571)=105 \mathrm{kgf} / \mathrm{cm} 2,
$$

that is much less than the design strength of the thin sheet steel

$$
R_{p}=2600 \mathrm{kgf} / \mathrm{cm} 2 .
$$

The bending at the first part at the middle of the three-layer plate span can be found due to the expression (6).

$$
(w)_{x=l / 2}=\frac{q l^{4}}{192 H^{2} D}\left(5+48 \omega+24 \frac{X_{1}}{q l^{2}}\right) .
$$

Taking into consideration the expression (30) for $X_{1}$, we obtain

$$
(w)_{x=l / 2}=\frac{q l^{4}}{192 H^{2} D}\left\{5+48\left[\omega-\frac{14}{265}\left(1-k_{8}^{(1)}\right)\right]\right\} .
$$

The relative bending at the middle of the first span of the three-layer plate is equal to

$$
\begin{aligned}
\frac{w}{l} & =\frac{q l^{3}}{192 H^{2} D}\left\{5+48\left[\omega-\frac{14}{265}\left(1-k_{8}^{(1)}\right)\right]\right\}= \\
& =\frac{0,016 \cdot 200^{3}}{192 \cdot 10^{2} \cdot 113187}(5+16,8-1,72)=\frac{1}{846} \leq\left[\frac{f}{l}\right]=\frac{1}{200} .
\end{aligned}
$$


The shear stresses in the aggregate are defined at the first part, where the upper-range value of the shear is located at the section from the first support to the left. It is calculated that

$$
\left(\tau_{z x}\right)_{\max }=0,5719 \frac{q l}{H}=0,5719 \frac{0,016 \cdot 200}{10}=0,18 \underset{\mathrm{kgf} / \mathrm{cm} 2<}{ } R_{c p}=0,4 \mathrm{kgf} / \mathrm{cm} 2 .
$$

\section{Conclusion}

The calculation results indicate the sufficient load-bearing capacity of the three-layer construction covering plate, which simultaneously satisfies the requirements imposed on it in respect of its deformability.

\section{References}

1. A.A. Zhuravlev, Architecture, 8, 56, (1966).

2. A.A. Zhuravlev, Ju.A Veselev, Higher Education News, Construction, 5-6, 26-30, (1993).

3. A.A. Zhuravlev, Higher Education News, Construction, 3, 19-24, (1994).

4. A.A. Zhuravlev, Ju.A.Veselev, An.A. Zhuravlev, Higher Education News, Construction, 3, 8-11, (1995).

5. K.Shtamm, H. Vitte, Multiply constructions (Strojizdat, Moscow, 1983).

6. V. Dundrová, V. Kovařík, P. Šlapák, Bigungstheorie der Sandwich-Platten. Academia. Verlag der Tschechoslowakischen Akademie der Wissenschaften (Praga, 1970).

7. Je.I. Grigoljuk, Izvestija AN SSSR, OTN, 1, 77- 84, (1957).

8. Je.I. Grigoljuk, P.P. Chulkov, Stability and vibrations of three-layered shells (Machine building, Moscow, 1973).

9. V.N. Kobelev, A.M. Kovarsky, S.I. Timofeev, Three-layer structures calculation (Machine building, Moscow, 1984).

10. V.F. Kulakov, J.V. Osetinskij, About the approximate calculation of three-layer structures taking into account the rheological properties of the aggregate (Rostov-onDon, 1974).

11. J.A. Veselev, Three-layer building structures (RGSU, Rostov-on-Don, 2013).

12. M. Langenbach, Bauen mit Holz, 11, 28-31, (2008).

13. V.I. Martemyanov, J.V. Osetinskij, Three-layer building structures (Rostov-on-Don, 1977)

14. J.V. Osetinskij, A.A. Shuravlov, H. Stenker, H. Bark, Zeitschrift der Jngenieurhochschulle Cottbus, 6, 7-16, (1984).

15. G. Hintersdorf, Tragwerke aus Plasten (VEB Verlag für Bauwesen, Berlin, 1972). 